\title{
Judged complexity as a function of schema related variables
}

SAM H. LANE AND SELBY H. EVANS, TEXAS CHRISTIAN UNIVERSITY, Fort Worth. Tex. 76129

The present study is an application of schema theory to complexity judgments. Three schemata, varying in regularity, and three levels of redundancy were combined to form nine conditions of stimulus complexity. The results indicate that the schema rule and redundancy level additively influence judged complexity.

Recent work in visual pattern perception has tended toward comprehensive specification and quantification of stimuli. The VARGUS 9 system of computer generated stimuli developed by Evans \& Mueller (1966) continued this trend. These patterns are sampled from a defined population with control of information and redundancy measures; the population is made up of variants which are independent and measurable deviations from a well-defined prototype or schema.

These patterns were originally designed for research on schema theory. The purpose of the present study was to extend the use of these patterns into another area of cognitive behavior, judged complexity. Other researchers have proposed that complexity is related to increases in the information content of patterns. Munsinger \& Kessen (1964) have defined complexity in terms of the number of independent turns in figures derived by an Attneave \& Arnoult (1956) procedure; information has been shown by Pollack \& Klemmer (1954) to be a logarithmic function of the number of independent turns. Vitz (1966) has defined complexity in terms of lines and angles which can be chained together in varying quantities to manipulate the information content of his patterns.

A potential advantage of VARGUS 9 patterns in research on judged complexity is that one can specify two parameters of complexity, both of which can be related to increasing bits of information. The first parameter relates to the simplicity of the schema or prototype. The second describes the average extent to which patterns deviate from the schema. The latter parameter has been termed schematic redundancy (Evans, 1967). The purpose of the present research was to manipulate judged complexity by independently varying the simplicity of the schema and the level of redundancy. It was hypothesized that judged complexity would increase additively as the schema was made more irregular and as redundancy was lowered.

Method. The Ss were 44 college students. The VARGUS 9 system produces output which can be printed as histoform patterns with column heights ranging from one to nine units. In this study, a unit corresponded to two characters on the printing device. The patterns were 21 columns in length.

A schema is introduced into the patterns by assigning to each column an average height. The VARGUS 9 program then produces patterns which deviate from the schema by the addition to each column height of a random variable, $d$, which is unimodally and symmetrically distributed about zero. Redundancy is manipulated by altering the probability distribution of $d$. (Details are given by Evans \& Mueller, 1962.)

Three schemata of differing degrees of irregularity were constructed as follows: Schema I was made completely regular by assigning all columns an average height of five. Schema II was made less regular by modifying Schema I; five columns were selected at random and each was assigned a randomly chosen average height other than five. Schema III was made still less regular by the same procedure used to produce Schema II, except that 10 columns of Schema I, instead of five, were changed. Redundancy levels of $40 \%, 50 \%$ and $60 \%$ were chosen on the basis of previous research with these patterns.

The design was a simple analysis of variance with repeated measures. (The design might also have been conceived as a two way analysis of variance with the two ways of manipulating complexity as crossed variables. No interaction was expected, however, and the primary interest lay in the relationship between increasing disorder and judged complexity.) The nine different combinations of schema rule and redundancy were treated as defining nine levels of stimulus uncertainty. Eighteen patterns were generated to exemplify each of the nine conditions. The eighteen patterns in each condition were then randomly assigned to three pages of groups of six patterns. This grouping was done to allow the $S$ to judge a sample from the schema family rather than individual patterns. The pages of patterns were randomly ordered into booklets of 27 pages each.

The Ss were instructed that the stimuli had been produced by means of a computer which had also assigned a "complexity" value to each group. Their task was to "match wits with the computer," and to rate each group of patterns in terms of its complexity. They were told to consider each page as a group, and to rate the group rather than any individual pattern. The Ss were allowed to spread out all the pages on a table and to inspect them before making the ratings.

The Ss were provided with a separate sheet of paper, numbered from one to 27 to correspond with numbers on the booklet pages. There were seven blank spaces on a line with each number. The blank to the extreme left was labeled "Least Complex," and the blank to the extreme right was labeled "Most Complex." The S was told to find the line corresponding to each group's number, and to place a check mark in the space which represented his complexity rating for that group. No additional instructions were provided as to the meaning of complexity. Responses were scored by assigning scale values of 1 through 7 to the seven spaces which corresponded to Least Complex through Most Complex. A S's score for each condition was his average rating of the three groups of patterns from that condition.

Results. The scores were subjected to a one-way analysis of variance with repeated measures (Winer, 1962) and a highly significant effect was found $(F=120.81$, df $=43, p<.01)$. A trend analysis on the means of the nine conditions indicated that $97.8 \%$ of the treatment variance could be accounted for by a linear component. Figure 1 presents the results graphically. It can be seen that only slight deviations from linearity appear between the interval of Schema I, $40 \%$ - Schema II, $60 \%$; and between the interval of Schema II, $40 \%$ - Schema III, $60 \%$.

Discussion. The additive nature of the influence of redundancy level and the generating rule upon judged complexity has been rather firmly established within the range of complexity as used in the present experiment. At more extreme levels of redundancy, a schema by redundancy interaction would be expected. From Fig. 1 it can be seen that "plateaus" in rating scores occur between Schema I, 40\% - Schema II, 60\%, and Schema II, 40\% - Schema III, $60 \%$. One possible explanation for these plateaus is that these two sets of

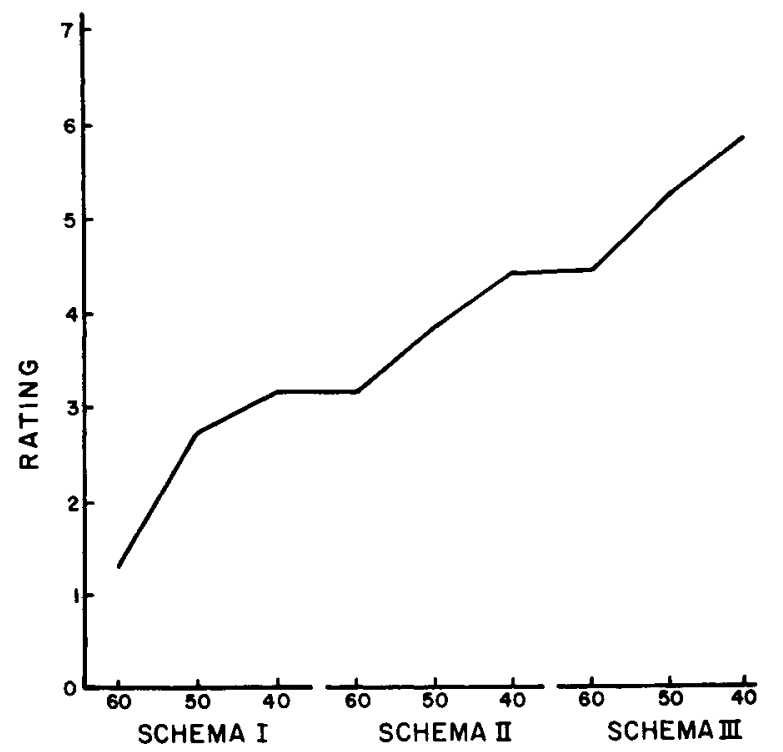

Fig. 1. Mean ratings $(N=44)$ of judged complexity across the nine manipulations of schema complexity. 
conditions are equal in subjective complexity. Another possible explanation is that the Ss are learning the schemata and the lowest level of redundancy within each schema is serving as an anchor point. Future research with a greater range of redundancy levels should clarify this point.

\section{REFERENCES}

ATTNEAVE, F., \& ARNOULT, M. D. The quantitative study of shape and pattern perception. Psychol. Bull., 1956, 53, 452-471.

EVANS, S. H. Redundancy as a variable in pattern perception. Psychol. Bull., 1967a, 67, 104-113.

EVANS, S. H., \& MUELLER, M. R. Vargus 9: computed stimuli for schema research. Psychon. Sci., 1966, 6, 511-512.

MUNSINGER, H., \& KESSEN, W. Uncertainty, structure, and preference. Psychol. Monogr., 1964, 78, 1-24.
POLLACK, I., \& KLEMMER, E. T. The assimilation of visual information from linear dot patterns. Technical Report No. 54-16, 1954, Air Force Cambridge Research Center.

VITZ, P. C. Preference for different amounts of visual complexity. Behav. Sci, 1966, 11, 105-114.

WINER, B. J. Statistical principles in experimental design. New York: McGraw-Hill, 1962.

\section{NOTE}

1. This research was supported in part by a Rehabilitation Service Administration training grant RSA-RH2 to the Institute of Behavioral Research and by Department of Defense Project THEMIS contract number DAAD05-68-C-0176 to T. C. U.

\section{Erratum}

Eastman, R. The relative crossmodal transfer of a form discrimination. Psychon. Sci., 1967, 9 (4), 197-198. The second sentence in the results section should read: "The means of the T-V and V-T groups, 5.20 and 3.54 respectively, showed that the $T-V$ sequence produced significantly superior transfer of the shape discrimination than did the V-T sequence." 